\title{
In silico insight into EGFR treatment in patients with lung carcinoma and T790M mutations
}

\author{
SHU-ZHI ZANG, YAN-RONG YANG, SHA-SHA ZHAO, YUN-XIA LI, XIN-YUAN GAO and CHUN-LEI ZHONG \\ Department of Respiration, The First Affiliated Hospital of Xinxiang Medical University, Weihui, Henan 453100, P.R. China
}

Received August 14, 2015; Accepted December 19, 2016

DOI: $10.3892 /$ etm.2017.4168

\begin{abstract}
The T790M mutational basis of treatment failure, following treatment via alteration of the epidermal growth factor receptor (EGFR) pathway, is a well-known anomaly in patients with non-small cell lung cancer (NSCLC). The T790M mutation activates the kinase domain, causing tyrosine kinase inhibitors, such as gefitinib, to elicit little or no response. To overcome this acquired resistance in NSCLC cells, the present study utilized a structure-based drug designing method to identify a novel lead compound. An in-house traditional Chinese medicinal compound database was used and following initial virtual screening, pre-absorption, distribution, metabolism and excretion/Tox and automated docking analyses, nardosinon was selected as the most appropriate candidate for further analysis. Two NSCLC cell lines, PC9GR4 and H2347, were used to test nardosinon and the results were compared with gefitinib. Results from an initial cell death assay revealed that nardosinon was able to induce cell death in NSCLC cells with and without the T790M mutation. These findings suggest that nardosinon may be an effective pharmacological compound for NSCLC treatment, including T790M EGFR mutant NSCLC cells.
\end{abstract}

\section{Introduction}

Non-small cell lung cancer (NSCLC) is the leading cause of cancer-associated mortality in both men and women worldwide (1). In China, there has been a drastic increase in lung cancer-associated mortality since 1970 (2). At present, treatments for NSCLC are marginally effective (3) and the primary target for treatment of NSCLCs is the epidermal growth factor receptor (EGFR) pathway (4). Other than genetic factors, patient characteristics including gender, race and lifestyle influence the effectiveness of the treatment for NSCLC (5). The most

Correspondence to: Dr Shu-Zhi Zang, Department of Respiration, The First Affiliated Hospital of Xinxiang Medical University, 88 Jiankang Road, Weihui, Henan 453100, P.R. China

E-mail: zangshuzhi@hotmail.com

Key words: T790M mutation, docking, Traditional Chinese Medicine, lung carcinoma prominent genetic factor associated with the responsiveness to NSCLC treatment is a mutation in EGFR $(6,7)$. Mutations in EGFR mediate the carcinogenic effect by manipulating the apoptotic mechanism (8). Tyrosine kinase inhibitors (TKIs) are the first line of treatment of cases featuring mutated EGFR (9). The initial response to TKIs is limited by acquired resistance and the T790M mutation in EGFR has previously been demonstrated to have this acquired resistance (10). A previous study revealed that the role of the T790M mutation is associated with an increase in the affinity of adenosine triphosphate (ATP) to mutated EGFR (11). Naturally derived compounds are being developed to treat NSCLC featuring a T790M mutation (12). Furthermore, computer-aided drug design has become a prominent tool in drug discovery (13). In the present study, an in-house Traditional Chinese Medicine (TCM) database was used to investigate novel drugs for EGFR treatment of NSCLC featuring this T790M mutation. In silico virtual screening tools, absorption, distribution, metabolism and excretion (ADME)/Tox analysis, and automated docking were all used to identify an effective single TCM compound.

\section{Materials and methods}

Protein preparation. The crystallographic structure of the kinase domain of the EGFR protein was retrieved from the Worldwide Protein Data Bank (PDB; wwpdb.org; ID:1XKK) (14). The structure was cleared of water and other ions and a T790M mutation was added using Discovery Studio Visualizer (Release 3.5.'; Accelrys, Inc., San Diego, CA, USA). The mutated structure was subjected to energy minimization using SPDB viewer version 4.1 (Swiss institute of Bioinformatics, Lausanne, Switzerland) following a previously documented protocol (15). GROMOS force field was used for the energy minimization.

Virtual screening. An initial dataset of 3,000 in-house selected TCM compounds, identified as exhibiting high activity, was used for the present study. This dataset was subjected to analysis with AutoDockVina version 1.1.2 (Scripps Research Institute, La Jolla, CA, USA) (16) platform using a Pymol interface (version 1.4.1; DeLano Scientific, Portland, OR, USA) (17). A grid of $40 \AA$ was created around the ATP binding site. The number of compounds was limited on the basis of Gibbs free energy $(\Delta G)$. Only those compounds with an $\Delta \mathrm{G}<-10 \mathrm{kcal} / \mathrm{mol}$ were selected for further ADME/Tox analysis. 


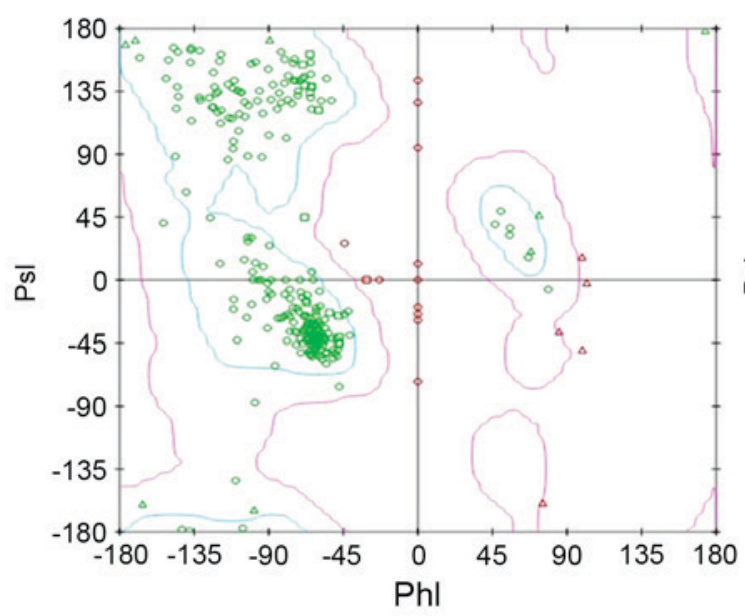

Before energy minimization

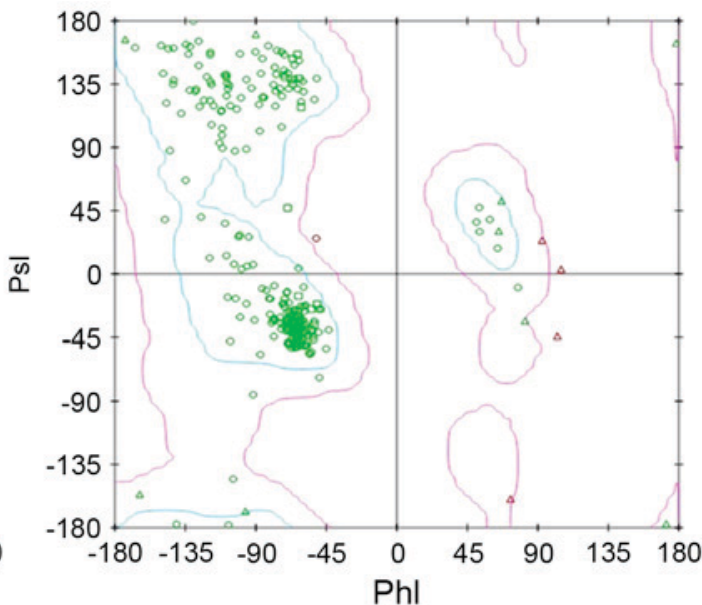

After energy minimization

Figure 1. The PsI-PhI distribution plot prior to and following energy minimization using GROMOS force field.

PreADME/Tox. An online sever (preadmet.bmdrc.org/) was used to evaluate the ADME of 25TCM compounds. The 4 TCM compounds, nardosinon, artesunate, daidzin and emetine were selected based on their ADME properties. The toxicity testing provided the mutagenicity and carcinogenicity properties of the selected compounds and only those compounds with no mutagenic and carcinogenic properties were selected for ADME evaluation. The predictive in silico ADME values and drug-likeliness values were used to further shortlist the compounds. Properties including molecular weight, the octanol/water partition coefficient $(\log \mathrm{P})$, number of hydrogen bond donors (HBD), number of hydrogen bond acceptors (HBA) and total polar surface area (tpsa) were taken into account.

Automated docking. The 4 most appropriate compounds, as determined by their ADME properties, were subjected to automated docking by AutoDock version 4.2 (Scripps Research Institute) (18). The Lamarckian algorithm of the tool was used to evaluate the optimal TCM compound for binding to the ATP binding pocket of EGFR. A total of 4 independent docking experiments were performed for the limited TCM compounds with maximum evaluation criteria based on number of runs for generating the suitable pose. The optimum generated pose was studied using LIGPLOT $^{+}$ software version 4.5.3 (European bioinformatics institute, Hinxton, UK).

Drug treatment and cell proliferation assay. The human lung adenocarcinoma cell lines PC9GR4 and H2347 were purchased from the American type culture collection (ATCC, Manassas, VA, USA) and used in the present study. Cells were seeded at a concentration of $1.5 \times 10^{4}$ cells $/ \mathrm{ml}$ in a 24-well plate and cultured at $37^{\circ} \mathrm{C}$ in an incubator containing $5 \% \mathrm{CO}_{2}$ for $24 \mathrm{~h}$ in Dulbecco's modified Eagle's medium supplemented with $10 \%$ fetal bovine serum (Gibco; Thermo Fisher Scientific, Inc., Waltham, MA, USA). Concentrations of nardosinon and gefitinib (Sigma-Aldrich; Merck Millipore, Darmstadt, Germany) ranging from 0.001 to $10 \mu \mathrm{M}$ dissolved in dimethylsulfoxide (DMSO), were subsequently added. The control group was treated with $0.1 \%$ DMSO alone (vehicle control). Each experiment was performed five times independently at each concentration. Cells were incubated at $37^{\circ} \mathrm{C}$ in a humidified atmosphere containing $5 \% \mathrm{CO}_{2}$ for $24 \mathrm{~h}$. The medium was subsequently removed and $0.1 \mathrm{mg} / \mathrm{ml}$ MTT solution (Sigma-Aldrich; Merck Millipore) was added to the cells followed by incubation for $4 \mathrm{~h}$ at $37.8^{\circ} \mathrm{C}$ in the dark. For control, 0.1 MTT solution was added to a plate containing no cells. The supernatant was subsequently removed and an equal volume of DMSO was added to dissolve the formazan crystals. The absorbance was measured at $565 \mathrm{~nm}$ against background absorption at $650 \mathrm{~nm}$ using an EPOCH Microplate Reader (BioTek Instruments, Inc., Winooski, VT, USA).

Statistical analysis. One-way analysis of variance was used to calculate significance. P-values between 0.001 and 0.01 were considered to indicate a significant difference. All statistical tests were performed using SPSS 18.0 for Windows (SPSS, Inc., Chicago, IL, USA).

\section{Results and Discussion}

Protein preparation and virtual screening. The T790M mutant form of EGFR kinase domain was prepared from crystallographic structure (PDB ID: 1XKK). The structure was subjected to energy minimization using GROMOS force field with SPDB Viewer, and PsI-PhI distributions prior to and following the energy minimization are depicted in Fig. 1. The purpose of virtual screening was to limit the number of compounds without affecting the success rate of compounds reaching further stages of drug development. A total of $25 \mathrm{TCM}$ compounds met the cut-off criteria $(\Delta \mathrm{G}<-10 \mathrm{kcal} / \mathrm{mol})$ set in virtual screening.

Predictive in silico ADME. The subset of 25 TCM compounds was further screened on the basis of their drug-likeliness according to Lipinski's rule of five (19). The online ADME/Tox tool was used to check Lipinski's parameters and provided an insight into the mutagenic and oncogenic potential of the compounds tested, parameters that are an important basis for 
Table I. Druglikeness and toxicity prediction.

\begin{tabular}{lccccccc}
\hline TCM No. & MW & $\log P$ & HBD & HBA & tpsa & Mutagenicity & Carcinogenicity \\
\hline 00812 & 384.178 & 3.10 & 1 & 8 & 101 & NO & NO \\
01273 & 416.111 & 0.46 & 5 & 9 & 146 & NO & NO \\
01578 & 480.229 & 4.49 & 1 & 6 & 52.2 & NO & NO \\
02108 & 250.333 & 2.96 & 1 & 3 & 35.5 & NO \\
\hline
\end{tabular}

Data generated using Pre ADME/Tox online tool. TCM, Traditional Chinese Medicine; MW, molecular weight; logP, octanol/water partition coefficient; HBD, number of hydrogen bond donors; HBA, number of hydrogen bond acceptors; tpsa, total polar surface area.

Table II. Auto Dock analysis of four lead natural products.

\begin{tabular}{|c|c|c|c|c|}
\hline Name & TCM No. & $\Delta \mathrm{G}, \mathrm{kcal} / \mathrm{mol}$ & Ligand binding pocket & Hydrogen bonds \\
\hline Lead 1 & 02108 & -6.38 & $\begin{array}{l}\text { ASN771, GLN791, VAL774, LYS852a, } \\
\text { ARG776 }{ }^{a} \text { MET790, LEU778, ALA1013, } \\
\text { ASP1014, LEU1017, LYS846 }{ }^{\text {a }} \text { PRO772 }\end{array}$ & $\begin{array}{l}\text { LYS852:N-: TCM:O }:_{2}(2.82 \AA) \\
\text { RG776:N-: TCM:O } \mathrm{O}_{3}(3.18 \AA) \\
\text { LYS846:N-: TCM:O } \mathrm{O}_{2}(3.15 \AA)\end{array}$ \\
\hline Lead 2 & 00812 & -5.82 & $\begin{array}{l}\text { ALA743, LEU792, VAL726, LEU844, } \\
\text { LEU718, MET766, PHE856, CYS775, LEU777, } \\
\text { LEU858, THR854, ASP855, ARG841 }\end{array}$ & NIL \\
\hline Lead 3 & 01273 & -1.32 & $\begin{array}{l}\text { ALA743, VAL726, LEU792, LEU718, } \\
\text { MET793, LEU777, LEU844, THR854 }^{a}, \\
\text { CYS755 }^{\text {a MET766, PHE856 }} \text {, LEU858, } \\
\text { ASP855 a , MET790, ILE789, LEU788 }\end{array}$ & 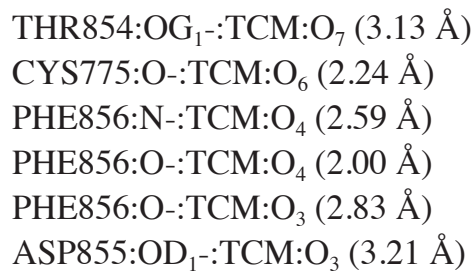 \\
\hline Lead 4 & 01578 & -0.32 & $\begin{array}{l}\text { VAL726, ALA743, ILE744, LYS745, } \\
\text { LEU788, LEU777, MET790, MET766, } \\
\text { ASP855, CYS755, THR854, PHE856, } \\
\text { GLN791, LEU792, MET793, LEU844 }\end{array}$ & NIL \\
\hline
\end{tabular}

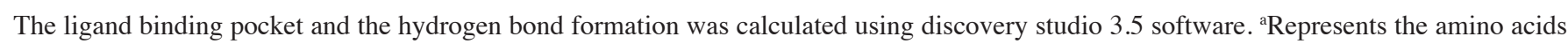
involved in forming hydrogen bond with the ligand. TCM, Traditional Chinese Medicine.

modern drug design. A total of 4 compounds were shortlisted and used for automated docking analysis. The following drug-likeliness parameters were used for the shortlisted compounds: Total polar surface area, molecular weight, calculated octanol/water partition coefficient, number of hydrogen bond donors and number of hydrogen bond acceptors (Table I).

Molecular docking analysis. The shortlisted TCM compounds were docked using the AutoDock 4.2 tool into the optimized adenosine triphosphate (ATP) binding site of the mutated EGFR protein and the results were analyzed using Pymol and $\mathrm{LIGPLOT}^{+}$software. The results generated by the in silico analysis are presented in Table II. All 4 shortlisted TCM compounds (Fig. 2) interacted with the ATP binding site via non-covalent interactions (hydrogen bonding). The two dimensional representation of the TCM-EGFR interactions (hydrophobic or non covalent) are presented in Fig. 3. The compounds were ranked based on the spontaneity of their interaction with EGFR. Nardosinon was determined to have a $\Delta \mathrm{G}$ of $-6.38 \mathrm{kcal} / \mathrm{mol}$ and form 3 hydrogen bonds with the
LYS852, ARG776 and LYS846 of the EGFR ATP binding pocket (Fig. 4A). The hydrophobic interaction pocket EGFR with nardosinon is comprised of the following amino acids: ASN771, GLN791, VAL774, MET790, LEU778, ALA1013, ASP1014, LEU1017 and PRO772. Oxygen at the second and third positions of the ligand interacts with LYS 852, LYS 846 and ARG776. The strongest interaction was between $\mathrm{N}$ on LYS852 and $\mathrm{O}_{2}$ on nardosinon, with a distance of $2.82 \AA$.

The second ranked TCM was artesunate, which interacted with the ATP binding pocket of mutated EGFR only. This binding pocket comprises ALA743, LEU792, VAL726, LEU844, LEU718, MET766, PHE856, CYS775, LEU777, LEU858, THR854, ASP855 and ARG841, and has a $\Delta \mathrm{G}$ of $-5.82 \mathrm{kcal} / \mathrm{mol}$ (Fig. 4B).

The third ranked TCM was daidzin, which was demonstrated to have a $\Delta \mathrm{G}$ of $-1.32 \mathrm{kcal} / \mathrm{mol}$. The binding pocket daidzin with mutated EGFR comprises the following amino acids: ALA743, VAL726, LEU792, LEU718, MET793, LEU777, LEU844, THR854, CYS755, MET766, PHE856, LEU858, ASP855, MET790, ILE789 and LEU788. Of these, 
A

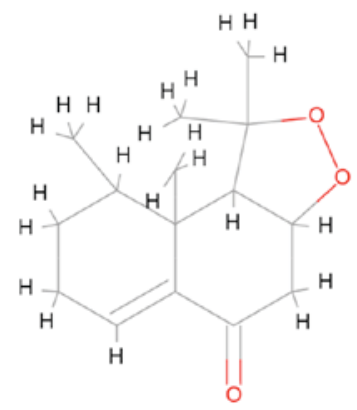

$\mathrm{C}$

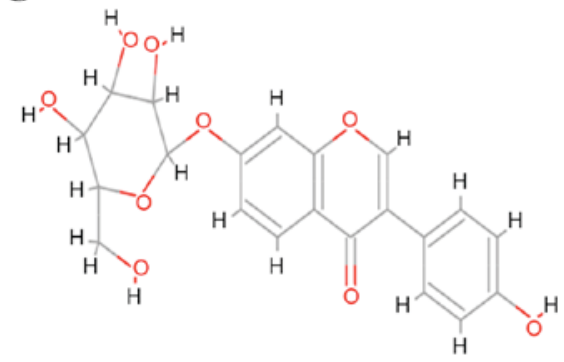

B

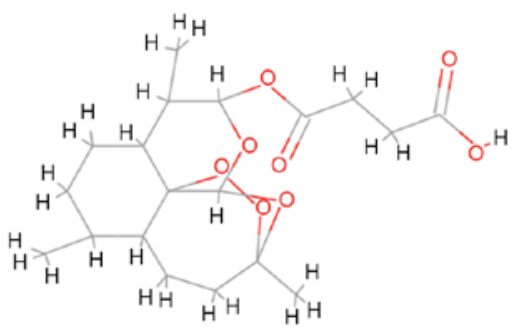

$\mathrm{D}$

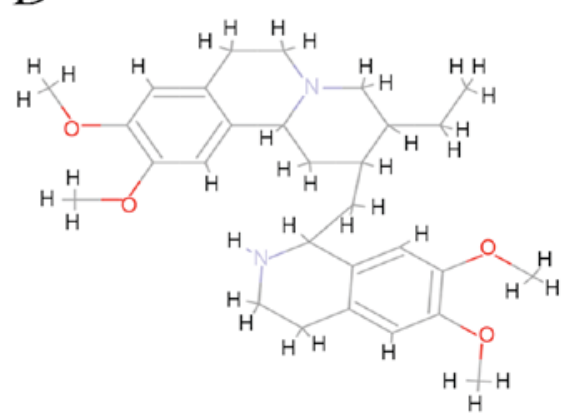

Figure 2. A total of 4 compounds were shortlisted from the Traditional Chinese Medicine database following virtual screening and absorption, distribution, metabolism and excretion/Tox analysis. (A) Nardosinon, (B) artesunate, (C) daidzin and (D) emetine.
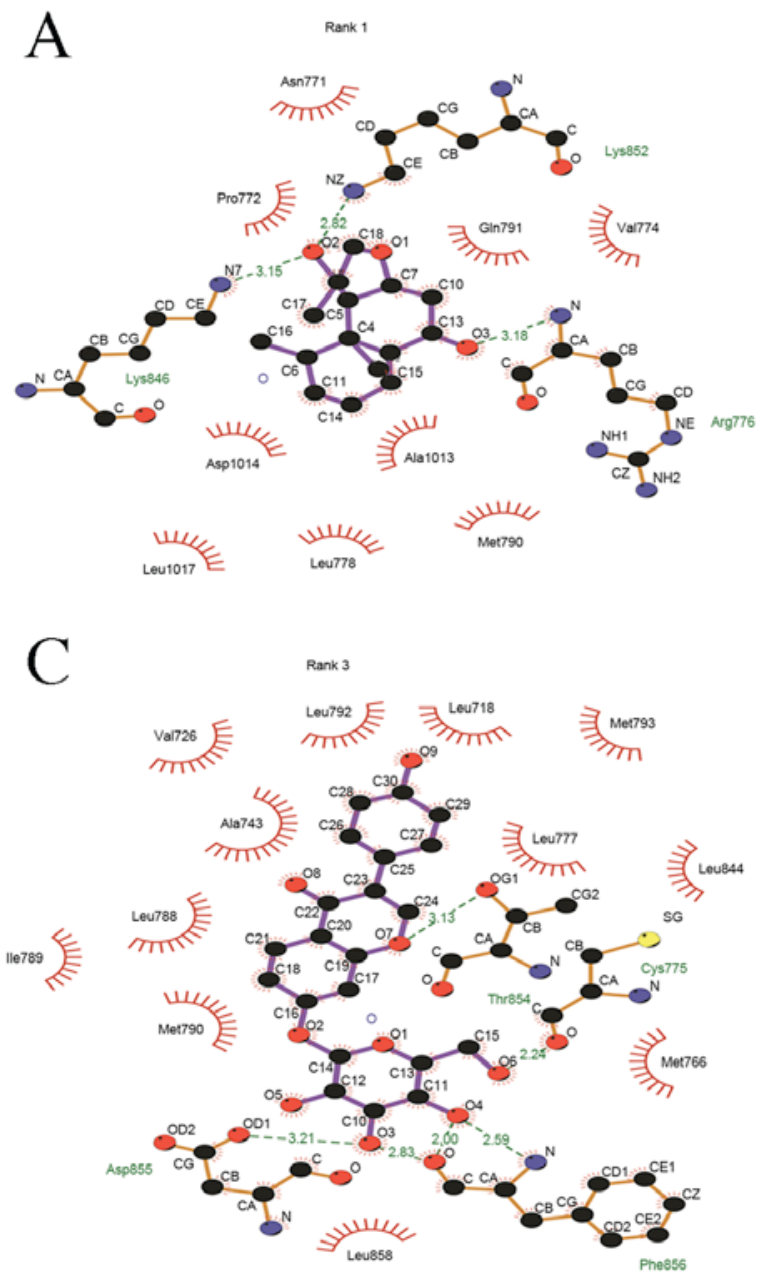

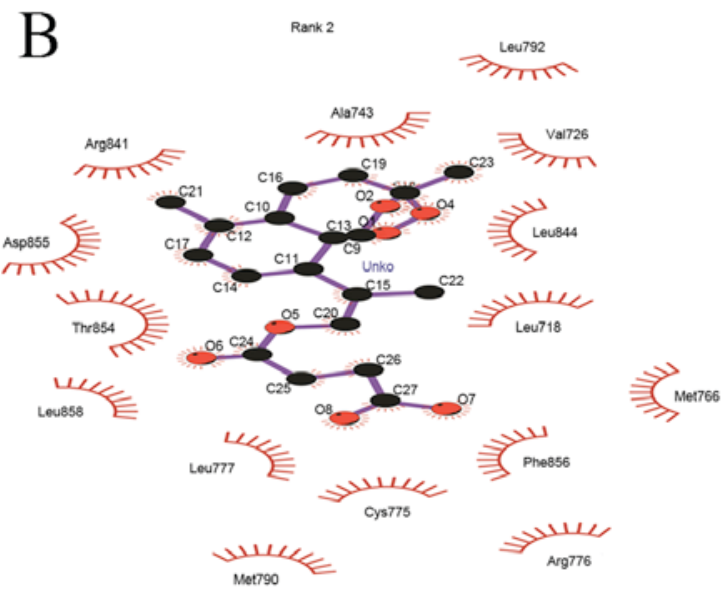

$\mathrm{D}$

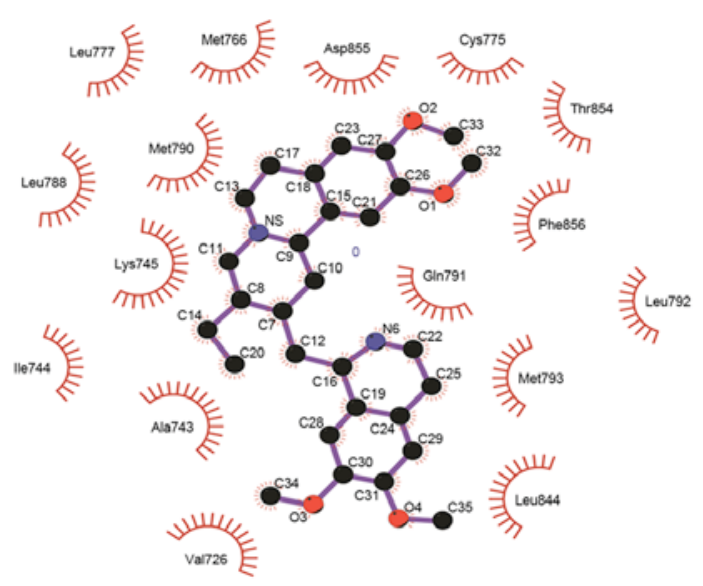

Figure 3. Two-dimensional contact plot depicting the noncovalent and hydrophobic interactions of (A) nardosinon, (B) artesunate, (C) daidzin and (D) emetine with the kinase domain of T790M mutated epidermal growth factor receptor. 
A

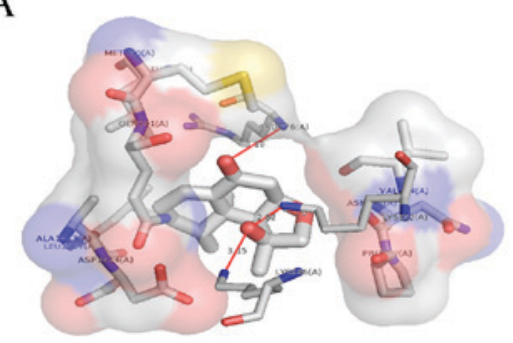

$\mathrm{C}$

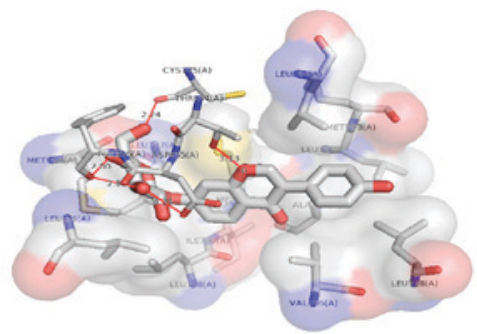

$\mathrm{B}$

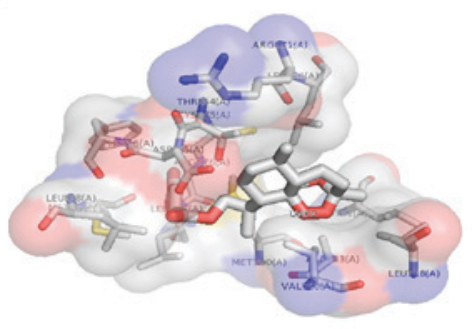

$\mathrm{D}$

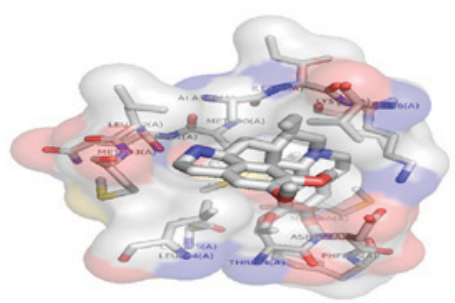

Figure 4. (A-D) Three-dimensional representation of atomic interactions. Red lines depict hydrogen bonds. (A) Nardosinon and (C) daidzin form hydrogen bonds, whereas (B) artesunate and (D) emetine exhibit contacts with hydrophobic pockets.

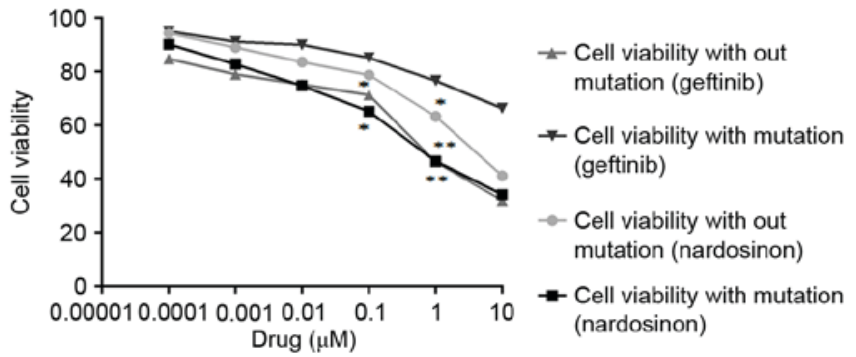

Figure 5. Inhibition of the EGFR kinase domain in mutated (PC9GR4) and non-mutated (H2347) cell lines by nardosinon at various concentrations $(0.1-10 \mu \mathrm{M})$. Cell viability was measured using crystal violet staining, following culture in 5\% fetal bovine serum with $100 \mathrm{ng} / \mathrm{ml} \mathrm{EGFR}$ at $72 \mathrm{~h}$ and exposure to indicated drug concentrations. Each data point represents the mean of 5 samples. Data represented as mean \pm standard deviation of results obtained from five independent experiments. ${ }^{* * *} \mathrm{P}<0.01 ;{ }^{* * * * *} \mathrm{P}<0.001$ compared with control. EGFR, epidermal growth factor receptor.

daidzin forms hydrogen bonds with 4 amino acids: THR854, CYS755, PHE856 and ASP855. The O atoms at the 3rd, 4th, 6 th and 7 th positions of daidzin form hydrogen bonds. Fig. $4 \mathrm{C}$ provides a 3D illustration of the interactions.

The fourth ranked TCM compound was emetine, which formed no non-covalent bonds with the mutated EGFR ATP binding domain. The hydrophobic binding pocket comprises the following amino acids: VAL726, ALA743, ILE744, LYS745, LEU788, LEU777, MET790, MET766, ASP855, CYS755, THR854, PHE856, GLN791, LEU792, MET793 and LEU844. The binding energy of the complex is $-0.32 \mathrm{kcal} / \mathrm{mol}$. Fig. 4D illustrates the hydrophobic interactions between emetine with the ATP binding domain.

Drug treatment and cell proliferation assay. To investigate the effect of the top ranked TCM compound, nardosinon, under in vitro conditions, the PC9GR4 cell line was used. These cells are derived from PC9 cells and are resistant to gefitinib treatment due to the T790M mutation. Cells were treated



Figure 6. Physical interaction of nardosinon with the kinase domain of the EGFR. Inset shows the pocket where binding of the compound from the Traditional Chinese Medicine database with the mutated EGFR occurs. EGFR, epidermal growth factor receptor.

with nardosinon at 5 different concentrations $(0.001,0.01,0.1$, 1 and $10 \mu \mathrm{M})$. A total of five independent experiments were performed for the 72-h assay with the different concentrations of nardosinon. The procedure was repeated for the $\mathrm{H} 2347$ cell line, representing NSCLC with wild type EGFR. Fig. 5 illustrates the cell viability (\%) vs. drug concentration of the two cell lines with and without the T790M mutation. The compound of interest, nardosinon (at a concentration $>0.1 \mu \mathrm{g}$ ) had a significantly greater effect compared with geftinib on the viability of cells with the T790M mutation $(\mathrm{P}<0.01)$, indicating that it could overcome the drug resistance caused by the T790M mutation in NSCLC patients. Following $72 \mathrm{~h}$ treatment, an IC50 of $225 \mathrm{mg} / \mathrm{ml}$ was calculated via MTT assay.

The T790M mutation is in the protein kinase domain of the EGFR and activates the ATP affinity of the EGFR kinase (20). Blocking this activity may have therapeutic potential. The 
difference in physical and chemical properties, including size, charge, and hydrophobicity value caused by the amino acid change from threonine to methionine at codon 790 , increased the activity in the kinase domain. In the present study, computer-aided drug design of a pharmacological agent for the treatment of patients with NSCLC and the T790M mutation has revealed a potentially effective TCM compound, nardosinon. Nardosinon is a compound found in the root extract of Nardostachys jatamansi (21). This plant is abundant in China and has a long history of medicinal use (22-25). Nardosinon is able to bind to the ATP binding site of the EGFR (Fig. 6) and the results from the in vitro studies of the nardosinon compound in two different cell lines indicates that it is may be developed as a pharmacological agent capable of treating NSCLC in patients with T790M EGFR variation.

\section{References}

1. Kobayashi S, Boggon TJ, Dayaram T, Jänne PA, Kocher O, Meyerson M, Johnson BE, Eck MJ, Tenen DG and Halmos B: EGFR mutation and resistance of non-small-cell lung cancer to gefitinib. N Engl J Med 352: 786-792, 2005.

2. Wang J, Jiang Y, Liang H, Li P, Xiao H, Ji J, Xiang W, Shi JF, Fan YG, Li L, et al: Attributable causes of cancer in China. Ann Oncol 23: 2983-2999, 2012

3. Scagliotti GV, De Marinis F, Rinaldi M, Crinò L, Gridelli C, Ricci S, Matano E, Boni C, Marangolo M, Failla G, et al: Phase III randomized trial comparing three platinum-based doublets in advanced non-small-cell lung cancer. J Clin Oncol 20: 4285-4291, 2002.

4. Mendelsohn J and Baselga J: Status of epidermal growth factor receptor antagonists in the biology and treatment of cancer. J Clin Oncol 21: 2787-2799, 2003.

5. Miller VA, Kris MG, Shah N, Patel J, Azzoli C, Gomez J, Krug LM, Pao W, Rizvi N, Pizzo B, et al: Bronchioloalveolar pathologic subtype and smoking history predict sensitivity to gefitinib in advanced non-small-cell lung cancer. J Clin Oncol 22: 1103-1109, 2004.

6. Wu YL, Zhou C, Hu CP, Feng J, Lu S, Huang Y, Li W, Hou M, Shi JH, Lee KY, et al: Afatinib versus cisplatin plus gemcitabine for first-line treatment of Asian patients with advanced non-small-cell lung cancer harbouring EGFR mutations (LUX-Lung 6): An open-label, randomised phase 3 trial. Lancet Oncol 15: 213-222, 2014.

7. Gervas P, Ivanova A, Vasiliev N, Ananina O, Zharkova O, Rogovieva O, Verzhbitskaya N, Didichuk I, Cheremisina O, Popova N, et al: Frequency of EGFR mutations in non-small cell lung cancer patients: Screening data from west siberia. Asian Pac J Cancer Prev 16: 689-792, 2015.

8. Chao TT, Wang CY, Lai CC, Chen YL, Tsai YT, Chen PT, Lin HI, Huang YC, Shiau CW, Yu CJ and Chen KF: TD-19, an erlotinib derivative, induces epidermal growth factor receptor wild-type nonsmall-cell lung cancer apoptosis through CIP2A-mediated pathway. J Pharmacol Exp Ther 351: 352-358, 2014.

9. Linardou H, Dahabreh IJ, Bafaloukos D, Kosmidis P and Murray S: Somatic EGFR mutations and efficacy of tyrosine kinase inhibitors in NSCLC. Nat Rev Clin Oncol 6: 352-366, 2009.

10. Helena AY, Arcila ME, Rekhtman N, Sima CS, Zakowski MF, Pao W, Kris MG, Miller VA, Ladanyi M and Riely GJ: Analysis of tumor specimens at the time of acquired resistance to EGFR-TKI therapy in 155 patients with EGFR-mutant lung cancers. Clin Cancer Res 19: 2240-2247, 2013.
11. Gandhi J, Zhang J, Xie Y, Soh J, Shigematsu H, Zhang W, Yamamoto H, Peyton M, Girard L, Lockwood WW, et al: Alterations in genes of the EGFR signaling pathway and their relationship to EGFR tyrosine kinase inhibitor sensitivity in lung cancer cell lines. PLoS One 4: e4576, 2009.

12. Fan XX, Wong MP, Cao ZW, Li N, Wu JL, Zhou H, Jiang Z, Liu L and Leung E: Abstract 3193: Apoptotic effect of a single compound derived from natural product in Gefitinib-resistant non-small cell lung cancer cells. Cancer Res 74: 2014.

13. Chikan NA, Bhavaniprasad V, Anbarasu K, Shabir N and Patel TN: From natural products to drugs for epimutation computer-aided drug design. Appl Biochem Biotechnol 170: 164-175, 2013.

14. Wood ER, Truesdale AT, McDonald OB, Yuan D, Hassell A, Dickerson SH, Ellis B, Pennisi C, Horne E, Lackey K, et al: A unique structure for epidermal growth factor receptor bound to GW572016 (Lapatinib) Relationships among protein conformation, inhibitor off-rate, and receptor activity in tumor cells. Cancer Res 64: 6652-6659, 2004

15. Guex N and Peitsch MC: SWISS-MODEL and the Swiss-PdbViewer: An environment for comparative protein modeling. Electrophoresis 18: 2714-2723, 1997.

16. Trott $\mathrm{O}$ and Olson AJ: AutoDock Vina: Improving the speed and accuracy of docking with a new scoring function, efficient optimization, and multithreading. J Comput Chem 31: 455-461, 2010.

17. Seeliger D and de Groot BL: Ligand docking and binding site analysis with PyMOL and Autodock/Vina. J Comput Aided Mol Des 24: 417-422, 2010.

18. Morris GM, Huey R, Lindstrom W, Sanner MF, Belew RK, Goodsell DS and Olson AJ: AutoDock4 and AutoDockTools4: Automated docking with selective receptor flexibility. J Comput Chem 30: 2785-2791, 2009.

19. Lipinski CA, Lombardo F, Dominy BW and Feeney PJ: Experimental and computational approaches to estimate solubility and permeability in drug discovery and development settings. Adv Drug Deliv Rev 46: 3-26, 2001.

20. Yun CH, Mengwasser KE, Toms AV, Woo MS, Greulich H, Wong KK, Meyerson M and Eck MJ: The T790M mutation in EGFR kinase causes drug resistance by increasing the affinity for ATP. Proc Natl Acad Sci USA 105: 2070-2075, 2008.

21. Bae GS, Kim MS, Park KC, Koo BS, Jo IJ, Choi SB, Lee DS, Kim YC, Kim TH, Seo SW, et al: Effect of biologically active fraction of Nardostachys jatamansi on cerulein-induced acute pancreatitis. World J Gastroenterol 18: 3223-3234, 2012.

22. Li W, Shi J, Li Q, Duan HH and Tang MK: Nardosinone reduces neuronal injury induced by oxygen-glucose deprivation in primary cortical cultures. Yao Xue Xue Bao 48: 1422-1429, 2013 (In Chinese).

23. Ju SM, Lee J, Kang JG, Jeong SO, Park JH, Pae HO, Lee GS, Kim WS, Lyu YS and Jeon BH: Nardostachys chinensis induces granulocytic differentiation with the suppression of cell growth through p27(Kip1) protein-related G0/G1 phase arrest in human promyelocytic leukemic cells. Pharm Biol 53: 1002-1009, 2015.

24. More SV, Koppula S, Kim IS, Kumar H, Kim BW and Choi DK: The role of bioactive compounds on the promotion of neurite outgrowth. Molecules 17: 6728-6753, 2012.

25. Ahmad A, Sattar MZ, Rathore HA, Fatima T, Khan SA, Afzal S, Lazhari M, Ahmed FU and Eseyin O: Pharmacological importance of Nardostachys jatamansi DC: A potential therapeutic agent in different pathological ailments. J Chem Pharmaceutical Res 5: 431-438, 2013. 